\title{
El Fenómeno sociodiscursivo de legitimación en el cubrimiento de las campañas de Carlos Gaviria y Álvaro Uribe durante las elecciones presidenciales del año 2006
}

\author{
The socio-discursive phenomenon of legitimisation in \\ the coverage of the campaigns of Carlos Gaviria and \\ Alvaro Uribe during the 2006 presidential elections
}

Raúl Enrique García López ${ }^{1}$

María Juliana Quintero Vélez ${ }^{2}$

Resumen

El presente artículo examina la representación mediática hecha por el diario El Tiempo de las campañas de los candidatos Álvaro Uribe y Carlos Gaviria en la elección presidencial del año 2006 en Colombia. En particular, este estudio analiza las formas como el fenómeno sociodiscursivo de legitimación, tal como la propone Pardo (2007), se manifestó en la representación que el diario hizo de los candidatos y sus campañas durante la semana previa a la elección. Mediante un análisis comparativo de los procesos lingüísticos de autorización y evaluación y mitigación, y su correlación con tres conceptos de la ciencia política tales como la dominación carismática (Weber, 1983), el bonapartismo (Moncayo, 2004) y la legitimidad (Lipset, 1981) se llega a conclusiones en cuanto al posicionamiento del diario en relación a las dos candidaturas. Se concluye que El Tiempo favoreció la campaña uribista mediante la representación carismática de su figura política y la legitimación de su proyecto político. De manera opuesta, el diario invisibilizó la figura de Carlos Gaviria y representó su proyecto como opuesto a los intereses de la mayoría del país.

Palabras clave:

legitimidad, elecciones, prensa escrita, Análisis Crítico del Discurso [ACD].

Abstract

This article examines how the daily newspaper El Tiempo represented the campaigns of the candidates Alvaro Uribe and Carlos Gaviria in the 2006 Colombian presidential election. In particular, this study analyses strategies such as the socio-discursive phenomenon of legitimisation, proposed by Pardo (2007), which manifests itself in the newspaper's representation of the candidates and their campaigns during the week before the election. By means of a comparative analysis of the linguistic processes of authorisation, evaluation and mitigation, and their correlation with three concepts in political science such as charismatic authority (Weber, 1983), Bonapartism (Moncayo, 2004) and legitimacy (Lipset, 1981), conclusions are reached regarding the stance adopted by the newspaper in terms of the two candidates. It is concluded that El Tiempofavoured Uribe's campaign because of the charismatic representation of his political figure and the legitimisation of his political project. In contrast, the newspaper made the figure of Carlos Gaviria invisible and represented his project as contrary to the interests of the majority of the country.

Key words:

legitimacy, elections, the press, Critical Discourse Analysis.

1 Universidad Industrial de Santander. Correo electrónico: raulspot3@gmail.com

2 Illinois State University. Correo electrónico: mjquinterov@gmail.com 


\section{Introducción}

Los medios de comunicación se pueden constituir como agentes no solo discursivos sino también políticos. Los medios son uno de los principales espacios en donde los ciudadanos se informan y comprenden la realidad sociopolítica de su país. Dadas las constricciones de espacio y tiempo a las cuales están sometidos los medios de comunicación, estos seleccionan los eventos de la realidad que constituyen el núcleo de lo informable. De esta manera, los medios desempeñan un papel crucial en la configuración de lo que se considera fundamental en la realidad política, social, económica de un país. Así, la realidad mediática viene a ser poco más que la reproducción de una determinada interpretación que un medio realiza de un evento. Esta reconstrucción está determinada por factores intrínsecos en los modelos mentales, representaciones sociales e ideologías imperantes en el sujeto comunicador, y por las estructuras de poder en el que está inmerso. (Fairclough, 1989, p. 50)

Teniendo en cuenta lo anterior, el presente artículo analiza la manera como la representación mediática redunda en la legitimación (o no) de los proyectos políticos. En particular, este trabajo estudia el papel que desempeñó el diario El Tiempo en las elecciones presidenciales del año 2006 en Colombia, mediante la representación que realizó de los dos principales candidatos y de sus proyectos políticos. Para la consecución de este objetivo, el Análisis Crítico del Discurso [ACD] representa una herramienta clave para el entendimiento de estas representaciones y, en el caso de esta investigación, para descifrar cómo se legitimaron o deslegitimaron los proyectos políticos más importantes en la carrera presidencial del 2006 en Colombia.

El caso colombiano merece particular atención debido a que tradicionalmente el país solo ha contado con dos diarios de circulación nacional: $E l$ Tiempo y El Espectador. Sin embargo, entre los años 2001 y 2008, El Espectador se transformó en semanario dejando a El Tiempo un implícito monopolio informativo. Este monopolio constituye una magnífica oportunidad para estudiar el rol de la prensa escrita en la (re)presentación de los hechos que dominaron la escena política durante este periodo.
En este escenario, el ámbito político colombiano presenció un hecho sin precedentes en la historia moderna del país: la posibilidad del presidente de aspirar a la reelección inmediata. Como consecuencia, Álvaro Uribe fue reelecto para el periodo 20062010 lo cual generó reacciones diversas en el ámbito político, especialmente debido a una serie de eventos que ocurrieron de manera simultánea: el proceso de negociación con los paramilitares así como los comprobados vínculos entre estas fuerzas ilegales y sectores políticos y económicos del país (fenómeno conocido por la opinión como la "parapolítica"); el advenimiento y consolidación de fuerzas opositoras de izquierda democrática; y la consolidación de un proyecto político de derecha materializado en la presidencia de Álvaro Uribe durante el periodo 2002-2010.

El presente trabajo constituye un esfuerzo interdisciplinar para el análisis de la representación mediática del discurso y los actores políticos orientado a aportar elementos de análisis que permitan entender el papel de la prensa escrita en la configuración de estos acontecimientos. Desde la ciencia política se adoptan conceptos como la legitimidad de los regímenes políticos (Lipset, 1981), la dominación carismática (Weber, 1983) y el bonapartismo (Moncayo, 2004). En términos del análisis lingüístico, se hace uso de la propuesta hecha por Pardo (2007). En particular se utilizará lo que ella denomina fenómeno sociodiscursivo de legitimación.

El corpus que constituye el objeto de esta investigación corresponde a los artículos publicados por el periódico El Tiempo durante la semana inmediatamente anterior a las elecciones presidenciales del 28 de mayo de 2006. Dado que el tamaño de la muestra no es representativo de la totalidad de la campaña, los resultados acá presentados no deben ser generalizados, y las conclusiones se refieren solo al cubrimiento realizado durante esta semana. La razón de tal selección está relacionada con la importancia que dicha semana tiene dentro del proceso electoral y la manera como los candidatos intensifican sus campañas y su presencia en los medios de comunicación durante la misma. Estos artículos constituyen 
el componente más importante del cubrimiento que el diario hizo de las elecciones y fueron publicados en la sección "Nación", sub-sección "Elecciones". Dentro de esta sección, solo se tomó en cuenta la información concerniente a Álvaro Uribe y Carlos Gaviria, quienes representaban las principales corrientes políticas en disputa.

\section{Marco teórico}

Se utilizaron tres ejes centrales para el análisis del corpus: los medios de comunicación como herramientas para el uso y/o abuso del poder (Van Dijk, 1997; 2004); la metodología para el análisis crítico del discurso propuesta por Pardo (2007); y la legitimidad del régimen político (Lipset, 1981), la dominación carismática (Webber, 1983) y el bonapartismo en la institución presidencial. (Moncayo, 2004)

Estas categorías ayudan a entender la manera como el fenómeno sociodiscursivo de legitimación propuesto por Pardo se hizo presente en los textos informativos del diario y si este fenómeno favoreció o no los proyectos políticos en cuestión.

\section{Los medios de comunicación como actores discursivos y políticos y su papel en la formación de opinión pública}

Los medios de comunicación influyen en la construcción de sentido, la cultura, lo vigente, lo aceptado, lo legítimo, etc.; sin embargo, es probable que una de sus funciones sociales más importantes sea su rol en la orientación de la opinión pública política, que adquiere especial importancia en los sistemas democráticos. Desde la perspectiva de Van Dijk (1997, p. 17), el papel de los medios en la opinión pública está estrechamente relacionado con el poder por las siguientes razones:

1. El discurso de información pública aporta las condiciones principales para la reproducción y la construcción del conocimiento en la mayoría de culturas y sociedades industrializadas. 2. Dicho conocimiento no es "objetivo", sino "sesgado", en el sentido de que está vinculado a los intereses de grupos de poder [...] 3. Las organizaciones mediáticas participan en esta estructura de poder [...] reproduciendo [...] este sesgo de conocimiento
El Fenómeno sociodiscursivo de legitimación en el cubrimiento de las campañas de Carlos Gaviria y Álvaro Uribe durante las elecciones presidenciales del año 2006.

Raúl Enrique García López, María Juliana Quintero Vélez

[...] 4. No obstante, este proceso de reproducción, no está siempre libre de dificultades. 5. En lugar de "transmitir" las creencias dominantes directamente, los medios de comunicación construyen una estructura interpretativa [...] es decir [...] no solamente delimitan las fronteras sino que también aportan el material de construcción para el consenso público, y de este modo fijan las condiciones [...] para [...] una hegemonía ideológica. 6 . No obstante, el proceso de producción ideológica no es unilateral [...] 7. [...] los medios de comunicación son parte integrante de la estructura de poder dominante [...] Al mismo tiempo, los medios de comunicación pueden actuar como agentes dialogantes entre los diversos, y a veces opuestos, grupos de poder.

De igual manera, Sartori (2003, p. 38) otorga un lugar constituyente a los medios en la democracia debido a que éstos tienen como responsabilidad informar al demos, que está a cargo de la toma de decisiones políticas. El acceso a la información es entonces fundamental para la opinión que pueden tener los ciudadanos y, en consecuencia, para la actuación política de estos a través de los diversos mecanismos de participación.

Es posible concluir, entonces, que las posiciones políticas ciudadanas no son una mera materialización de las posturas ideológicas individuales, sino que están constituidas por la negociación entre estas posturas, los valores sociales y la influencia que los medios ejercen en la difusión de los hechos políticos. Un ejemplo de cómo la massmediación ha transformado la escena política es la manera en que ésta se ha convertido en el lugar principal de debate y captación de simpatizantes, reemplazando a la tradicional plaza pública. Esta nueva realidad, según Martín-Barbero (1997, p. 10) introduce modificaciones sustanciales en cómo los ciudadanos representan mentalmente la política y en las formas en que estos se organizan y desarrollan mecanismos de acción y representación democrática.

\section{El fenómeno sociodiscursivo de legitimación en la representación mediática}

Una vez comprendido el papel de los medios en la estructuración de la opinión pública, el ACD se 
constituye en una alternativa para analizar la configuración de las dinámicas de control social en los discursos mediáticos. En este sentido, Pardo (2007, p. 121) propone dos niveles de análisis. El primero está relacionado con un análisis textual que permite llegar a conclusiones en cuanto a la frecuencia en el uso de ciertas palabras, frases, expresiones etc., y la manera como éstas se correlacionan, generando la consolidación de unidades de significado en torno a un elemento del discurso. El segundo es un análisis de tipo cualitativo que estudia el discurso desde el nivel sintáctico, pasando por las estrategias discursivas y llegando a un nivel pragmático en donde se encuentran los fenómenos sociodiscursivos, socioculturales y sociopolíticos. En la Tabla 1 se presenta el mencionado análisis.

Como podemos observar, el nivel de análisis más general encierra un fenómeno sociopolítico, el cual se denomina exclusión-inclusión. Este se puede dar a través de dos fenómenos socioculturales, naturalización y ocultamiento; los cuales, a su vez, se (re) presentan por medio de tres fenómenos sociodis- cursivos: consistencia-coherencia, legitimación y transformación. En cada uno de estos se presentan diversas estrategias discursivas y procesos lingüísticos. Esta investigación se concentra en el fenómeno sociodiscursivo de legitimación, en cuanto está relacionado con la legitimidad del régimen político. Dentro de la propuesta de Pardo, el proceso de legitimación sociodiscursiva se da a través de las estrategias de persuasión, negociación, acusación y justificación, las cuales se desarrollan a través de cinco procesos lingüísticos: autorización, racionalización, evaluación, narrativización y mitigación.

En la coyuntura tratada, el fenómeno sociodiscursivo de legitimación es trascendental. Para las elecciones presidenciales del año 2006, el gobierno de Álvaro Uribe impulsó una reforma constitucional para permitir su propia reelección, lo cual había sido prohibido por la Carta Magna de 1991. Por esta razón, el periodo previo a la elección fue vulnerable a la intención de las élites cercanas al presidente de legitimar su acciones con el objetivo de alcanzar la reelección y así verse favorecidas en sus intereses,

Tabla 1. Propuesta de análisis cualitativo para el ACD.

\begin{tabular}{|c|c|c|c|c|}
\hline $\begin{array}{l}\text { Fenómeno } \\
\text { Socio-Político }\end{array}$ & $\begin{array}{l}\text { Fenómeno } \\
\text { Socio-Cultural }\end{array}$ & $\begin{array}{l}\text { Fenómeno Socio- } \\
\text { Discursivo }\end{array}$ & $\begin{array}{l}\text { Estrategias } \\
\text { Discursivas }\end{array}$ & $\begin{array}{l}\text { Procesos Lin- } \\
\text { gǘsticos }\end{array}$ \\
\hline \multirow{3}{*}{$\begin{array}{l}\text { Inclusión- } \\
\text { Exclusión }\end{array}$} & \multirow{2}{*}{ Naturalización } & $\begin{array}{l}\text { Consistencia- } \\
\text { Coherencia }\end{array}$ & $\begin{array}{l}\text { Segmentación } \\
\text { Integración } \\
\text { Ambivalencia }\end{array}$ & $\begin{array}{c}\text { Tematización } \\
\text { Focalización } \\
\text { Citación/Noces }\end{array}$ \\
\hline & & Legitimación & $\begin{array}{l}\text { Persuación } \\
\text { Negociación } \\
\text { Justificación }\end{array}$ & $\begin{array}{c}\text { Autorización } \\
\text { Racionalización } \\
\text { Evaluación } \\
\text { Narrativización } \\
\text { Mitigación * }\end{array}$ \\
\hline & Ocultamiento & Transformación & $\begin{array}{l}\text { Elisión } \\
\text { Reordenamiento- } \\
\text { Sustitución }\end{array}$ & $\begin{array}{c}\text { Supresión total } \\
\text { Supresión parcial } \\
\text { Activación- } \\
\text { Pasivación } \\
\text { Personalización- } \\
\text { Impersonalización }\end{array}$ \\
\hline
\end{tabular}

Tomada de Pardo (2007, p. 121).

* Énfasis propio de los autores para resaltar el enfoque metodológico del presente trabajo. 
configurando lo que Van Dijk (2004, p. 11) ha denominado abuso del poder.

\section{La legitimidad como bastión del régimen político}

La posibilidad de que una democracia sea estable está condicionada no solamente por su desarrollo económico, sino también por la efectividad y legitimidad de su sistema político. La efectividad es entendida como la medida en que las necesidades de sus ciudadanos son atendidas. Estas necesidades están determinadas por la ideología imperante en dicha sociedad. Así, por una parte, sociedades netamente capitalistas priorizan necesidades relacionadas con el libre mercado, la posibilidad de adquisición de bienes suntuarios, etc. Por otra parte, sociedades de tipo socialista asumen como necesidades aspectos relacionados con la igualdad y el acceso a derechos fundamentales.

En cuanto a la legitimidad del régimen político, se entiende como la convicción por parte de los ciudadanos de que la ideología imperante es la más adecuada. Tiene que ver con la manera como los ciudadanos no solo aceptan esta ideología sino que la hacen parte de su sistema de valores. La incorporación de estos valores se constituye en un proceso de control social el cual se lleva a cabo, en ocasiones, a través de las prácticas discursivas. Este proceso de legitimación de un régimen político dominante es fundamental en la estabilidad de los gobiernos. Lipset (1981, p. 106) habla de la manera como la efectividad y la legitimidad se relacionan para ser los bastiones de un régimen. Los gobiernos cuya legitimidad y efectividad se encuentran en un punto alto son altamente estables. De manera opuesta, aquellos con baja efectividad y legitimidad son naturalmente
El Fenómeno sociodiscursivo de legitimación en el cubrimiento de las campañas de Carlos Gaviria y Álvaro Uribe durante las elecciones presidenciales del año 2006.

Raúl Enrique García López, María Juliana Quintero Vélez

inestables. Empero, al observar combinaciones distintas en donde la legitimidad del gobierno es mayor que su efectividad, la estabilidad de este es mayor que la de aquellos gobiernos cuya efectividad es más alta que su legitimidad. En la Tabla 2 se refleja la relación entre efectividad y legitimidad y sus efectos en la estabilidad del régimen político.

En otras palabras, es más importante para la estabilidad del régimen que sus ciudadanos crean que su gobierno está ejecutando políticas adecuadas que la efectividad real de éstas. Por esta razón, los sistemas políticos se han visto obligados a expandir su campo de acción a esferas donde sea posible legitimar su ideología. Es aquí en donde los medios masivos de comunicación se constituyen no solo como mediadores entre la estructura de poder y el público, sino también como el escenario donde las ideologías se forman, se reproducen y se comercializan.

La legitimidad de un sistema político, entonces, encuentra un prolífico campo de acción en la esfera mediática. La propuesta de Pardo presenta un esquema transferible al estudio de la legitimación del sistema político, donde el proceso sociodiscursivo de legitimación se presenta como uno de los bastiones de la representación mediática.

\section{Dominación carismática y bonapartismo en Colombia}

El poder es ejercido legítimamente cuando existe una voluntaria sumisión por parte de los ciudadanos, la cual es resultado de un proceso de dominación. Según Weber (1983, p. 172) el poder se entiende como la posibilidad de un individuo o grupo de imponer su voluntad sobre la de los demás. Weber describe tres tipos de dominación legítima: la

Tabla 2. Estabilidad del régimen político.

\begin{tabular}{|c|l|l}
\hline Efectividad & \multicolumn{1}{|c|}{ Alta } & \multicolumn{1}{c}{ Baja } \\
\hline Alta & Estabilidad & Menor posibilidad de estabilidad \\
\hline Baja & Mayor posibilidad de estabilidad & Inestabilidad \\
\hline
\end{tabular}

Nota: "Efectividad y legitimidad de los regímenes políticos". En Lipset (1981). 
dominación legal, fundamentada en los reglamentos establecidos racionalmente que se aplican a través de la ley. La dominación tradicional, basada en virtud de la costumbre. Y la dominación carismática, en la cual la obediencia se deposita en la persona del jefe, lo que hace que esta sumisión no sea racional sino emocional. De acuerdo a la dominación carismática el líder es considerado poseedor de "una enorme capacidad de convocatoria [...] una especie de mesianismo que arrastra tras sí ciudadanos, envueltos en una mística que les transmite su jefe. El ciudadano así impregnado políticamente en un fervor cuasirreligioso..." (Blanco Ande, 1990, p. 332).

Durante años, el Estado colombiano ha vivido una crisis de estabilidad de su sistema político debido a su baja efectividad y legitimidad. En otras palabras, a pesar de ser consagrado como Estado de derecho, la presencia de un prolongado conflicto armado y los altos niveles de corrupción sugieren que Colombia no tiene la capacidad de ejercer una dominación legal debido a su inhabilidad de impartir justicia y sus niveles de impunidad. El caso colombiano tampoco se ajusta a la dominación tradicional porque la validez de la costumbre no es considerada inviolable en términos legales.

La dominación carismática parece ser la más cercana al régimen político colombiano. Este tipo de dominación hace referencia a las características individuales del gobernante, a su entrega personal, sacrificio e identificación con el pueblo. Como lo evidencia Palacios (2002), el caso de Álvaro Uribe es fácilmente asimilable a este concepto:

Se trata de un presidente a caballo [...] hombre antioqueño individualista, trabajador, tenaz, festivo, blanco y católico; amante de la autoridad y en primer lugar de las jerarquías católicas [...] el poder se ha encarnado en un representante típico de [...] los grandes hacendados, y por lo tanto, heredero de toda la carga de tradicionalismo que caracteriza a aquellos dueños de hacienda, esos verdaderos señores de la tierra, que son la sustancia de los poderes regionales y locales.

Lo anterior, la manera como se presenta cercano a la mayoría de la población colombiana y su omnipresencia en los asuntos del Estado, generó durante el periodo 2002-2006 una concentración del poder en su figura política, coincidiendo con lo que Moncayo (2004, p. 349) ha denominado como bonapartismo: "un sistema civil que asume misiones de orden, sobre bases personales de carácter carismático, superponiéndose a las funciones tradicionalmente reconocidas a otras ramas del poder y suspendiendo determinadas garantías ciudadanas o acudiendo a mecanismos militares y policivos".

Si se analiza desde la división de poderes de Montesquieu, la situación expuesta es problemática. Según este autor, el poder público no es uno solo, sino que se divide en tres: legislativo, ejecutivo y judicial. El régimen bonapartista diluye esta división del poder, concentrándolo en la figura del presidente y generando serias implicaciones para la administración del Estado y el poder (Burdeau, 1981, p. 139). El presidencialismo bonapartista de Uribe se ampara en su popularidad, su reputación de hombre serio y laborioso, y la asociación que de él se hace con valores tradicionales como la religión o la unidad familiar: "En la naturaleza irracional de una popularidad que lo convierte en salvador providencial de un país sin norte, el presidente muestra el corazón blando a quienes lo aplauden, reservándose la mano dura para quienes disienten" (Collazos, 2003).

\section{Marco Metodológico}

Para este estudio se ha analizado el fenómeno sociodiscursivo de legitimación a través de tres procesos lingüísticos: autorización, mitigación y evaluación (Pardo, 2007, p. 121). En primer lugar, se rastrean los procesos lingüísticos en el corpus y se determina su concurrencia con las categorías políticas planteadas así como con los candidatos en cuestión. En segundo lugar, se desarrolla una matriz descriptiva (ver Tabla 3) que permite visualizar estas concurrencias en el caso de cada candidato. En tercer lugar, se realiza un análisis comparativo de la representación mediática de los dos candidatos. Se pretende establecer en qué medida las diferencias o similitudes se manifiestan en relación con las categorías políticas. En cuarto lugar, se plantea la manera como se 
Tabla 3. Matriz descriptiva de los textos informativos.

\begin{tabular}{l|l|l|l|}
\hline $\begin{array}{l}\text { Fecha: } \\
\text { Subtitular candidato: }\end{array}$ & Titular principal: \\
\hline & Proceso Linguístico & Mitigación & Autorización \\
\hline Categoría Política & Evaluación & \\
\hline Figura Política & & \\
\hline Gasto Social & & \\
\hline Posicionamiento electoral & & \\
\hline
\end{tabular}

Nota. Esta matriz de análisis se aplicó a los artículos para establecer correlaciones entre las categorías políticas y las categorías lingüísticas. Tabla producto de los autores.

configura la exaltación y/o ocultamiento de la figura y campaña política de cada uno de los aspirantes a la presidencia. Finalmente, se presentan conclusiones con respecto a si se favoreció la legitimidad de alguna de las campañas políticas.

\section{Categorias para el análisis lingüístico}

El primer proceso lingüístico que se analiza es el de autorización en donde, al conceder voz discursiva a otros actores, el emisor argumenta, anecdotiza y/o trivializa lo que se afirma, y por lo tanto, legitima o deslegitima el sujeto del discurso. Los procesos de autorización involucran al actor discursivo externo al que se recurre y a lo dicho por éste. Estos textos pueden tener una connotación positiva o negativa. Así, es necesario establecer relaciones entre los predicados (lo que se está diciendo), los sujetos (de quién se está diciendo) y las voces discursivas que los referencian (quién lo está diciendo), con el fin de establecer si se trata de un proceso de legitimación o deslegitimación. En otras palabras, un mismo pre- dicado expuesto por dos actores discursivos distintos puede favorecer o no al sujeto de ese predicado.

Por estas razones se propone una subclasificación de los procesos de autorización. Primero, la autorización positiva hace referencia a la manera en que, al apelar a actores discursivos externos (que presuponen una pertinencia y autoridad sobre la situación), se fortalece una afirmación con un sentido positivo sobre las características de un sujeto. En otras palabras, se legitima a un sujeto mediante un otro que habla por el medio y que da fuerza al argumento expuesto. Segundo, la autorización negativa hace referencia a la manera en que un medio se apoya en un actor discursivo externo para deslegitimar una afirmación relacionada con un acontecimiento o sujeto, y de esta manera evadir su responsabilidad en una eventual evaluación negativa del hecho informado. En la Tabla 4 se presentan ejemplos ${ }^{3}$ de los fenómenos de autorización positiva y negativa.

3 Todos los ejemplos son tomados del corpus de la investigación. Para mayor detalle ver lista de referencias.

Tabla 4. La autorización positiva y negativa.

\section{Autorización positiva}

"[...] Álvaro Uribe, el presidente más popular de todos los tiempos y quién seguramente será reelegido en primera vuelta este 28 de mayo -eso advierten los sondeos- no logró que sus seguidores colmaran la Plaza de Bolívar [...]".

\section{Autorización negativa}

"[El optimismo y entusiasmo del Polo] no es solo por el repunte en las encuestas del candidato Carlos Gaviria. Se debe, según él ${ }^{*}$, a la respuesta que encontró en la plaza pública remplazada desde hace varios años por los medios, sobre todo, por la televisión".

* El énfasis es propio de los autores para señalar la presencia de agentes discursivos externos. 
En el primer ejemplo, el diario recurre a un actor discursivo externo (los sondeos) para darle un velo de autoridad a la afirmación que hace (seguramente será reelegido en primera vuelta). Este es un caso de autorización que pretende legitimar lo afirmado por el medio acerca de Álvaro Uribe o, como se ha denominado, un caso de autorización positiva. De manera opuesta, en la segunda cita el diario recurre a una voz discursiva externa fácilmente interpretada como carente de objetividad, lo cual resta validez a lo que se informa (la respuesta que encontró en la plaza pública). Este es un caso de autorización negativa.

El segundo proceso lingüístico es la evaluación. Este consiste en la emisión de juicios de valor acerca de un evento o sujeto en particular, se observa la frecuencia en el uso de adjetivos y su carácter. En la cita referente a Uribe notamos la inclusión de una frase adjetiva con una alta connotación positiva (más popular de todos los tiempos), que modifica la frase nominal (Álvaro Uribe). El uso de este tipo de modificadores configura una unidad de significado favorable para la imagen del candidato. En este caso específico se está presentando a Uribe como el presidente más popular en la historia de Colombia. Sin embargo, se trata de una afirmación subjetiva, carente de evidencia alguna. La frecuencia de este tipo de procesos y su relación con los candidatos permite establecer si existe una exaltación de la figura de alguno de ellos.

El tercer proceso lingüístico es la mitigación. Éste consiste en la presentación de un hecho mediante una cuidadosa elección lexical, el uso de eufemismos, la configuración de la información presentada y el uso reiterado de los procesos arriba definidos. Un hecho que afecte o favorezca a un sujeto en particular puede ser presentado como un evento natural o una anécdota. Los procesos de mitigación encierran un intento por restarle o asignarle importancia a un evento de la realidad.

Una misma oración puede contener múltiples procesos discursivos. Como podemos ver en el ejemplo de autorización positiva descrito anteriormente en la tabla 4 , aunque los procesos de evaluación (el presidente más popular de todos los tiem- pos) y autorización (eso dicen los sondeos) están presentes y se pueden analizar de manera aislada, es posible concluir que éstos, desde un análisis integral del texto, están en función del proceso de mitigación. El artículo al que pertenece la cita en mención informa sobre el final de la campaña en la plaza pública. El candidato Uribe obtuvo una respuesta desfavorable que contrastaba con la gran acogida que obtuvo en las encuestas. Sin embargo, cada vez que el diario hace referencia a esta realidad recurre a procesos lingüísticos que la mitigan. De esta manera, los procesos de evaluación y autorización, de manera conjunta, atenúan la información negativa.

\section{Categorias para el análisis político}

El rastreo y análisis de los procesos lingüísticos se hace en relación con tres categorías políticas: la figura política, el gasto social y el posicionamiento electoral. Las correlaciones que se establecen con los candidatos permiten llegar a conclusiones en cuanto a lo que se legitima.

En primer lugar, la figura política es una categoría conceptual que hace referencia a un personaje representativo de un grupo o colectivo cuya orientación ideológica lo coloca en abierta disputa por el poder en una sociedad. Las figuras políticas pueden ser personajes representativos en los diversos ámbitos geográficos (local, departamental, nacional e internacional) en las tres ramas del poder público (ejecutiva, legislativa, judicial), en las funciones de control, en los procesos electorales, en los partidos políticos, en los grupos de interés, en los grupos de oposición y en la Iglesia, etc. Así, en el presente estudio la categoría de figura política se emplea para referirse a los candidatos Álvaro Uribe y Carlos Gaviria.

En segundo lugar, el gasto social da cuenta de las posiciones que tienen los distintos proyectos políticos frente al uso de los recursos estatales para la ejecución de las políticas públicas. Según Franco (1983) el gasto social puede ser percibido de cuatro maneras: 1) Se entiende como una carga, es decir, como un uso de los recursos públicos para el mantenimiento administrativo y funcional del estado y para garantizar el acceso de la población a los dere- 
chos fundamentales y servicios básicos. 2) El gasto se concibe como inversión en capital humano sano lo cual promueve el desarrollo económico. 3) Se encuentra el gasto social como complementario, en donde se impulsan actividades de un sector que logran servir para el desarrollo de otro. 4) El gasto puede ser entendido como medio de control social, al asignar y distribuir recursos a diferentes poblaciones vulnerables que después pueden constituirse en capital electoral.

Finalmente, el posicionamiento electoral es un proceso mediante el cual se establece la correlación de fuerzas entre los candidatos que participan en una contienda electoral. La categoría de posicionamiento electoral se concentra en dos ejes. Por una parte, están las campañas desarrolladas en la plaza pública a través de formas tradicionales de hacer política, en donde los líderes apelan a sus habilidades oratorias para convocar seguidores y establecer un contacto personal con ellos. Por otra parte, están aquellas campañas que utilizan herramientas como los medios impresos, electrónicos y los sondeos, las cuales se asocian con formas modernas de hacer política en donde los líderes aumentan su visibilidad ante sectores de la población alejados de la política.

\section{Resultados y Análisis}

El análisis del corpus permite identificar claras diferencias en la representación que hizo el diario de los dos candidatos en disputa, entre las cuales se encuentra la legitimación de Álvaro Uribe y su proyecto político. Esta legitimación se materializó en los tres procesos lingüísticos objeto de análisis en el corpus.
El Fenómeno sociodiscursivo de legitimación en el cubrimiento de las campañas de Carlos Gaviria y Álvaro Uribe durante las elecciones presidenciales del año 2006.

Raúl Enrique García López, María Juliana Quintero Vélez Representación de la campaña de Álvaro Uribe

El proceso de evaluación se hizo presente en la representación de la figura carismática de Uribe mediante el uso de frases adjetivas y verbales de carga semántica positiva. Uribe fue constantemente representado como una figura asociada con valores patrióticos y culturales. A continuación, presentamos las principales instancias en las cuales el diario recurre a este proceso. (Tabla 5).

Este fenómeno también se dio en la representación que el diario hizo de los escenarios donde se llevaron a cabo sus actos de campaña. Algunos ejemplos son:

Mujeres cabeza de familia, de piel morena y cuerpos macizos, con sus hijos prendidos a la cintura dando gracias a Uribe por los favores recibidos [...] para sus niñitos. [...] Con lenguaje de acordeoneros, guacharacas y tambores la Costa Atlántica [...] Uribe se despidió a las 6:15 pm, cuando el sol ya besaba el mar, con un ruego [...].

Mediante estos elementos, el diario representa a Álvaro Uribe como estandarte de la identidad cultural de la sociedad colombiana, legitimando su figura como el principal líder político del país y lo representa de una manera narrativizada y estética, recurriendo al detalle excesivo y a la inclusión de elementos lingüísticos innecesarios para el cubrimiento de lo informado. Igualmente, la legitimación de la figura de Uribe se realizó también de manera retrospectiva y la consolidación de su carácter bonapartista se evidenció mediante el proceso de autorización positiva. La confusión estratégica de rol en torno a las distintas maneras de nominalización

Tabla 5. El proceso de evaluación en la representación de Álvaro Uribe.

Proceso de Evaluación / Tipo de Representación

Candidato cercano al pueblo

Persona de gran carisma y de carácter popular

Padre y hombre de familia

Trabajador incansable

\section{Cita}

"Este paisa, que se convierte en costeño adoptado cuando pisa el nivel del mar, le respondió a los caribeños en su propio lenguaje."

"Uribe estuvo muy emotivo y coqueto con la ciudad..."

"Uribe llegó al Patio [sic] Mosquera [...] en compañía de su esposa Lina Moreno [...]; de sus hijos Tomás y Jerónimo (escoltados por sus novias)"

"él [Uribe] les devolverá cuatro años de trabajo intensivo, sin vacaciones ni días festivos" 
“presidente-candidato, presidente, etc.", otorgó al mandatario un lugar privilegiado de enunciación. La promoción de sus actos de gobierno en situaciones de campaña y el planteamiento de propuestas electorales en actos de gobierno generó una desigualdad en las garantías del proceso electoral. Esta ambigüedad genera que todo acto de gobierno sea percibido como acto de campaña y que los actos de campaña adquieran la potencial fuerza de actos de gobierno. En uno de los artículos referentes a las principales propuestas de los candidatos, la sección dedicada a Álvaro Uribe está caracterizada por la presentación de los bancos de oportunidades, haciendo alusión directa a los avances que, en materia de los créditos a bajo costo, se venían desarrollando en el gobierno. Aquí, el velo de autoridad lo tienen los programas sociales del régimen vigente, que hablan por el diario y por el presidente aspirante a su reelección:

Banco de Oportunidades: El gobierno habilitó 140 mil millones de pesos para fondear el Banco de las Oportunidades, creado por mandato presidencial hace dos semanas, para financiar con créditos de bajo costo, sin fiador, procesos de emprendimiento de los sectores más pobres. Los recursos de crédito deberán ser tramitados a través de intermediarios como la banca privada y oficial, algunas organizaciones sin ánimo de lucro y un nuevo agente que el propio presidente Álvaro Uribe bautizó como "corresponsales de pueblo".

El diario no menciona que los bancos de oportunidades fueron una de las propuestas de gobierno de Álvaro Uribe para desarrollar en la presidencia 2006-2010, sino que inicia describiendo lo que había venido haciendo como presidente. Esto denota una confusión estratégica de roles entre el candidato que argumenta sus propuestas, y el presidente que expone sus avances políticos de gobierno. Es posible entonces afirmar que esto introduce ambigüedad en el texto al presentar la propuesta de campaña como un programa social del régimen. Se dificulta inferir si el Banco de Oportunidades es una ejecución del mandato 2002-2006 o una propuesta para el periodo 2006-2010.

En relación con lo anterior, el diario imprime verosimilitud en la ejecución de las propuestas al mismo tiempo que visibiliza al agente ejecutor.
Se emplea para ello el uso de formas verbales que connotan un alto grado de posibilidad, "ampliará, hará, incorporará, etc.", todas atribuidas al sujeto "Uribe". A pesar del contexto de baja efectividad de las instituciones estatales, Uribe se muestra como un mandatario eficiente y autónomo, reforzando la concepción bonapartista del poder que promueve el periódico al desconocer la naturaleza tripartita del sistema político colombiano. La materialización de las propuestas se sujeta únicamente al hecho de que sea reelegido, desconociendo la importancia política del legislativo en el trámite de las demandas sociales.

En relación con el gasto social, vale la pena destacar que el diario destina una serie de artículos para cubrir, lo que a su juicio, son los principales temas de la coyuntura. Estos contienen, por un lado, "las tres preguntas clave" sobre distintos asuntos de interés político, y por el otro, información sobre el desarrollo de la campaña de cada candidato. Aquí, el diario se postula a sí mismo como vocero de la opinión pública que presenta los cuestionamientos y utiliza diversas estrategias para visibilizar/invisibilizar las propuestas y figuras de los candidatos. En el artículo que aborda el tema de salud tanto el sumario como el subtitular principal hacen referencia al tema del día, pero en el caso de la subtitulación de las partes concernientes a Uribe y a Gaviria solo aparece el nombre de Uribe: "Es dañino que Uribe no haya debatido" y "No sembrar mas arroz. Uribe". Ambas frases son citas directas que visibilizan su figura.

Adicionalmente, el diario recurrió al proceso de mitigación para informar aspectos de la campaña que eran claramente desfavorables para el candidato Uribe. En la Tabla 6 podemos evidenciar esto de manera más clara.

En los ejemplos se hacen evidentes dos regularidades. Primero, las expresiones que hacen alusión a la situación desfavorable para la campaña de Uribe (la ausencia de seguidores) son subordinadas, mientras que los procesos de evaluación positiva con información no relacionada con el evento informado son focalizados. Segundo, la inclusión de complementos que en todos los casos favorecen la imagen del candidato Uribe con expresiones emocionalmente cargadas como "arrasa", o "multitudes rabiosas que corean su nombre y le anuncian 
El Fenómeno sociodiscursivo de legitimación en el cubrimiento de las campañas de Carlos Gaviria y Álvaro Uribe durante las elecciones presidenciales del año 2006.

Raúl Enrique García López, María Juliana Quintero Vélez

Tabla 6. El proceso de mitigación en el candidato Uribe.

Hecho informado

Se presentó una asistencia inusualmente baja en los actos de campaña de Uribe

Se presentó una asistencia inusualmente baja en los actos de campaña de Uribe

Se presentó una asistencia inusualmente baja en los actos de campaña de Uribe

Se presentó una asistencia inusualmente baja en los actos de campaña de Uribe

Se presentó una asistencia inusualmente baja en los actos de campaña de Uribe

\section{Representación hecha por el diario}

"Uribe siempre ha sido recibido por multitudes rabiosas que corean su nombre, aplauden sus logros y le anuncian su respaldo. Pero en ciudades como Medellín, Cali, Manizales y Bogotá, los escenarios a los que concurrió estuvieron muy holgados de asistentes".

"Aunque quienes concurrieron a su encuentro siempre expresaron su fervor y su admiración por el mandatario, en varias de esas citas el número de asistentes no colmó las expectativas".

"Los choques admitidos por los uribistas han llevado a la contradicción de que el Presidente [sic] más popular de todos los tiempos, que arrasa en todas las encuestas y seduce por televisión, no logre llenar escenarios que Uribe quisiera ver abarrotados de seguidores".

"Las escasas concurrencias han sido achacadas a problemas de organización. A dificultades generadas por los celos de los jefes uribistas regionales, en ningún caso a falta de convocatoria del candidato Uribe".

"No parece que estos sean tiempos de gloria para la plaza pública, es, más bien, el de los medios electrónicos y las encuestas". su respaldo". El uso de estas dos estrategias termina por mitigar un hecho que no favorece la figura del posicionamiento electoral de Uribe.

\section{Representación de la campaña de Carlos Gaviria}

En el caso del candidato Gaviria el proceso que se presentó con mayor frecuencia fue el de mitigación. Pero en esta ocasión se utilizó para el efecto opuesto. (Tabla 7)

En el caso de Gaviria la buena acogida por parte de sus seguidores en la plaza pública es mitigada por medio de procesos de autorización negativa para ratificar que la plaza pública ya no es determinante para la opinión pública. Esta relevancia en los procesos electorales la poseen ahora los medios electrónicos y las encuestas: "No parece que estos sean tiempos de gloria para la plaza pública, es, mas bien, el de los medios electrónicos y las encuestas". Adicionalmente, la respuesta recibida en la plaza pública por Gaviria es mitigada por el uso de diversas estrategias, como el uso de comillas que trivializan los acontecimientos: "El 'optimismo' y 'entusiasmo' del Polo [...] Se debe, según él, a la respuesta que encontró en la plaza pública, un escenario que desde hace varios años había sido desplazado por los medios, y sobre todo, por la televisión"

Es importante detenernos a analizar esta oración en detalle. En ella encontramos al menos dos componentes principales. En primer lugar, una puesta en duda de lo que se afirma mediante un proceso de

Tabla 7. El proceso de mitigación en el candidato Gaviria.

\begin{tabular}{|l|l|}
\hline \multicolumn{1}{|c|}{ Hecho informado } & \multicolumn{1}{c}{ Representación hecha por el diario } \\
\hline Alta asistencia de personas al acto de campaña & $\begin{array}{l}\text { “El candidato del Polo señala que la 'alta asistencia’ de seguidores que se con- } \\
\text { centraron en Cartago y Buga [...] le permiten concluir que 'esta es la verdadera } \\
\text { encuesta”” }\end{array}$ \\
\hline Alta asistencia de personas al acto de campaña & $\begin{array}{l}\text { “El ‘optimismo y entusiasmo' [...] No es solo por el repunte en las encuestas del } \\
\text { candidato Carlos Gaviria. Se debe, según él, a la respuesta que encontró en la } \\
\text { plaza pública, un escenario que desde hace varios años había sido desplazado”. }\end{array}$ \\
\hline Alta asistencia de personas al acto de campaña & $\begin{array}{l}\text { "Dicen que Uribe está algo preocupado. En su gira por Pasto el candidato-presi- } \\
\text { dente le preguntó a su acompañante: 'QQué le estará diciendo Gaviria a la gente } \\
\text { que está creciendo tanto?"” }\end{array}$ \\
\hline
\end{tabular}


autorización negativa al usar la frase preposicional "según él". En segundo lugar, una nueva mitigación de la importancia de la plaza pública en los procesos electorales al afirmarse que esta ha sido "desplazada [...] por la televisión".

De la misma manera el proceso de mitigación se realiza a través de estrategias como la anecdotización de la realidad expuesta al afirmarse: "La estrategia tomó un nuevo aire en esta contienda por la Presidencia, pues también, dicen en el Polo, obligó al presidente a echar discursos al aire libre" para luego asegurar: "Dicen que Uribe está algo preocupado[...] en Pasto [...] le preguntó a un acompañante: ‘¿Qué le estará diciendo Gaviria a la gente que está creciendo tanto?"' En estas dos citas es posible encontrar varios elementos. Primero, una reiteración del cuestionamiento a la buena respuesta de los seguidores del candidato Gaviria así como a la reacción de su contendiente, mediante la inclusión de una cita indirecta y anónima en un proceso de autorización negativa; "dicen en el Polo". Segundo, un cuestionamiento del crecimiento del candidato al otorgarle voz discursiva a su oponente que se pregunta: “QQué le estará diciendo Gaviria a la gente que está creciendo tanto?" Tercero, la "supuesta" preocupación de Uribe no se presenta claramente sino que se introduce mediante un proceso de autorización negativa. No se cita fuente veraz, confiable o al menos reconocible, generando cierta incredulidad con respecto a la preocupación del candidato.

En otras palabras, el diario no presentó el éxito creciente de la campaña de Gaviria sino que, por el contrario, éste es cuestionado recurrentemente: "La pregunta de hoy, a 7 días de las elecciones es: ¿le alcanzará el impulso para forzar una segunda vuelta?". Así, sometiendo un evento de la realidad que a todas luces favorece al candidato de la oposición a un proceso de mitigación mediante el uso de las estrategias que cuestionan, trivializan, anecdotizan e individualizan esta favorabilidad; el diario pone en duda la posibilidad de que este hecho pueda influir de alguna manera en el desenlace de la coyuntura electoral.

Finalmente, en términos generales, la representación del posicionamiento electoral puede ser resumida en una unidad de significado compuesta por las siguientes proposiciones: La plaza pública ya no es importante para el proceso electoral; los medios electrónicos remplazaron a la plaza pública en la postulación del probable devenir del proceso electoral; y Uribe es imbatible en los medios electrónicos y las encuestas.

En cuanto a la representación de las propuestas de Gaviria, podemos afirmar que se dio un proceso de mitigación igualmente. En muchos casos en los que se citó a Gaviria para presentar sus propuestas programáticas, se recurrió a formas condicionales que denotaban un grado de probabilidad de realización bajo: "Y en cuanto al manejo de la deuda externa, buscaría su renegociación con la banca internacional", "Gaviria usaría los mecanismos de participación previstos en la Constitución de 1991". Este estilo de citación llama particularmente la atención al analizarlo de manera comparativa con la representación de las propuesta uribistas, las cuales siempre fueron citadas usando formas gramaticales con un alto grado de probabilidad "apoyará," "se comprometió", "presentará", "ofreció convertirse en fiador", “[...] cuya creación decidió[...]”, La encuesta pregunta: “[...] si están de acuerdo o no con el apoyo de las FARC a la candidatura de Gaviria”.

En otros casos se recurrió al proceso de autorización negativa para, mediante la citación de actores discursivos principalmente anónimos, relacionar a Gaviria con grupos ilegales: "Además dicen que Gaviria recibió el sábado pasado la adhesión a ese grupo [FARC] y citan un reporte de un noticiero de televisión: según el cual, en la frontera con Venezuela se habrían decomisado 15 mil millones de dólares camuflados [...]”.

Este tratamiento por parte del periódico opacó la imagen de Carlos Gaviria. El único aspecto que se menciona de este candidato como figura política es su condición de académico e intelectual. Además, la representación gráfica de Gaviria casi siempre se realiza en recintos cerrados, contextos aislados y sin presencia de seguidores. La figura política de éste y la importancia del proyecto político que representa se pierden en la nominación genérica que se realiza de la izquierda y del colectivo denominado como "la oposición". Este planteamiento se refuerza mediante la citación directa que hace el periódico del can- 
didato para criticar los actos políticos de Álvaro Uribe. Si se considera que este último se presenta como el líder del colectivo que representa al país, es fácil reconocer el carácter antagónico con el que $E l$ Tiempo representó a Gaviria.

Las referencias al gasto social en Gaviria son casi nulas, presentadas de manera nominalizada, excluyendo así el aspecto de causalidad, tal como lo propone Fairclough (1989, p. 51), “[...] construcción de un modelo de sociedad y de Estado que ponga fin a las injusticias sociales y las desigualdades [...]", “[...] la ley no da cubrimiento universal y para quienes tienen cubrimiento, un POS o sisbén, son muy mediocres". El Tiempo no presenta a Gaviria como proponente y ejecutor de estas propuestas, y cuando lo hace, utiliza formas verbales condicionales cuyo grado de verosimilitud es inferior al caso de la representación de las propuestas uribistas. Gaviria es presentado como un candidato antagonista y sin propuestas viables. Esto se hace manifiesto en la utilización que el diario hace del espacio destinado a la presentación de estas propuestas para hablar de temas como los rumores de fraude y las críticas de la oposición al oficialismo.

De esta manera vemos como el diario hace un uso diferencial de los procesos de evaluación, mitigación y autorización para legitimar el proyecto político de Álvaro Uribe y para invisibilizar la candidatura de Carlos Gaviria. Principalmente, el diario utilizó el proceso de evaluación para exaltar la figura carismática de Uribe, mientras que en el caso de Gaviria hubo una baja frecuencia en el uso de este proceso; en realidad fue prácticamente inexistente. En contraste, el proceso de mitigación se utilizó de manera recurrente en relación con los dos candidatos, principalmente en lo que concierne a su posicionamiento electoral. En el caso de Gaviria se utilizó para mitigar la respuesta positiva que encontró en los eventos en plaza pública, mientras que en el caso de Uribe se recurrió a éste para mitigar la importancia de la plaza pública en el proceso electoral y la inesperada baja respuesta que encontró en los eventos públicos. Por último, el proceso de autorización se utilizó principalmente, en el caso de Uribe, para validar su obra de gobierno y para
El Fenómeno sociodiscursivo de legitimación en el cubrimiento de las campañas de Carlos Gaviria y Álvaro Uribe durante las elecciones presidenciales del año 2006.

Raúl Enrique García López, María Juliana Quintero Vélez

visibilizar la importancia de los sondeos en el proceso electoral. En el caso de Gaviria, este proceso se utilizó para poner en tela de juicio ciertas realidades de la carrera electoral que favorecían su candidatura y para relacionarlo con grupos al margen de la ley y con sectores minoritarios.

\section{Conclusión}

La presente investigación constituye un esfuerzo interdisciplinar para analizar, a la luz del Análisis Crítico del Discurso [ACD], la forma como los medios de comunicación influyen en el devenir político de las sociedades. En el caso aquí presentado es posible concluir que el periódico El Tiempo fortaleció la dominación carismática como uno de los bastiones de la estabilidad del régimen político colombiano en la coyuntura, a través de un tratamiento diferencial de los dos candidatos a las elecciones presidenciales del 2006. En este sentido, el diario legitima el proyecto político del presidente del momento, Álvaro Uribe, y su permanencia en el poder mediante tres elementos: La exaltación de su figura carismática, la promoción de su concepción de gasto social a través de la exaltación de sus actos de gobierno como presidente en situaciones de campaña y la validación de las encuestas como un factor decisivo en el desenlace de las elecciones. Por el contrario, en el caso del candidato de la oposición, Carlos Gaviria, el periódico invisibilizó su imagen política y personal, así como sus propuestas políticas; y restó importancia a su posicionamiento favorable al deslegitimar el rol de la plaza pública en el proceso electoral.

Finalmente, vale la pena señalar algunas limitaciones del presente estudio y sugerir futuras rutas de investigación. A pesar del riguroso análisis realizado, la dimensión del corpus utilizado impide llegar a conclusiones más amplias. Teniendo en cuenta lo anterior, se sugiere para futuras investigaciones la inclusión de los dos procesos lingüísticos que no fueron tenidos en cuenta en el presente estudio (racionalización y narrativización), con el objetivo de proveer un análisis más amplio del fenómeno sociodiscursivo de legitimación, tal y como es propuesto por Pardo (2007). Adicionalmente, se reco- 
miendan muestras más grandes, tanto en la cantidad de información noticiosa como en la diversidad de medios, es decir, material adicional al proveniente de la prensa escrita (por ejemplo material de la televisión o internet). En este sentido, una muestra más completa puede ofrecer mayor cobertura, lo cual, con metodologías apropiadas, puede permitir estudios sobre el efecto directo que sobre la población tiene el discurso mediático y sus consecuencias en términos del comportamiento de los ciudadanos.

\section{Referencias}

El Tiempo (20 al 27 de mayo, 2006). Sección: Nación; Subsección: Elecciones.

Blanco, J. (1990). Teoría del poder. Madrid: Beramar.

Burdeau, G. (1981). Derecho constitucional e instituciones políticas. Madrid: Editorial Nacional Cultura y Sociedad Torregalindo.

Collazos, O. (12 de septiembre, 2003). Imprudencia, ira o demagogia. El Tiempo. Recuperado de http://www. eltiempo.com/archivo/documento/MAM-991368

Fairclough, N. (1989). Language and power. New York: Longman.

Franco, R. (1983). Las grandes controversias de las políticas sociales. Santiago de Chile: CEPAL, ILPES, UNICEF.

Lipset, S. M. (1981). El gobierno: Estudios comparados. Madrid: Alianza Editorial.

Martín-Barbero, J., Espinel, M. y Restrepo, A. (1997). La nueva representación politica en Colombia. Bogotá: IEPRI, Fundación Friedrich Ebert de Colombia.

Moncayo, V. M. (2004). El leviatán derrotado: Reflexiones sobre teoría del estado y el caso colombiano. Bogotá: Ediciones Norma.

Palacios, P. (7 de agosto, 2002). Un presidente "de a caballo". El País. Recuperado de http://elpais.com/diario/2002/08/07/internacional/1028671207_850215. html

Pardo, N. G. (2007). Cómo hacer análisis crítico del discurso. Santiago de Chile: Frasis Editores.

Sartori, G. (2003). Video política: Medios, información $y$ democracia de sondeo. Madrid: Fondo de Cultura Económica.

Van Dijk, T. (1997). Racismo y análisis crítico de los medios. Barcelona: Ediciones Páidos Ibérica.

Van Dijk, T. (2004). Discurso y dominación. Grandes Conferencias en la Facultad de Ciencias Humanas, 4.

Weber, M. (1983). Economía y sociedad. México: Fondo de Cultura Económica. 